\title{
A Hybrid Artificial Bee Colony for Robust and Coordinated Power System Stabilizer Design
}

\author{
Anderson Sampaio dos Santos \\ Graduate Program in Electrical \\ Engineering - PPEE \\ Universidade Federal de Juiz de Fora \\ Juiz de Fora, Brazil \\ andersonsampaio@yahoo.com \\ anderson.sampaio@estudante.ufjf.br
}

\author{
Wesley Peres \\ Department of Electrical Engineering, \\ Federal University of São João del-Rei \\ - UFSJ \\ São João Del-Rei, Brazil \\ wesley.peres@ufsj.edu.br
}

\author{
João A. Passos Filho \\ Department of Electrical Engineering, \\ Federal University of Juiz de Fora - \\ UFJF \\ Juiz de Fora, Brazil \\ joao.passos@ufjf.br
}

\begin{abstract}
This paper presents a hybrid optimization method to solve power system stabilizers' robust and coordinated problem. The tuning procedure is formulated as an optimization problem with the objective of maximizing closedloop damping coefficients considering various scenarios to ensure robustness. The proposed hybrid method combines the global search based on an Artificial Bee Colony (ABC) algorithm in food collection with the local search capability of the Quasi-Newton optimization method (Broyden - Fletcher Goldfarb - Shanno - BFGS). The method is successfully applied to the well-known New England test system and its results outperform the ones provided by conventional ABC, EPSO, PSO and Genetic Algorithm.
\end{abstract}

Keywords-Hybrid ABC, Power System Stabilizers, Robust and Coordinated Project, Quasi-Newton Method.

\section{INTRODUCTION}

Low-frequency electromechanical oscillations damping has been performed through Power System Stabilizers (PSS) since the 1970s [1]. Such undamped oscillations can cause mechanical wear on the generating units and blackouts and reduce the power transfer limits between areas. These originate from the electrical and mechanical torques unbalance in synchronous generators after disturbances [2].

PSS is a control structure allocated at the generator's excitation systems to provide damping torque [1]. The methods for stabilizers tuning can be divided into techniques based on robust control, classical control, and optimization techniques. Optimization methods work with explicit indices that quantify the closed-loop system performance. They are interesting because they allow the choice of an index to be optimized, and the project can be performed automatically with minimal designer interference [3].

There are many optimization methods (derivative-based approaches and metaheuristics) to be used in the PSS project. Analytical methods based on objective function derivatives have the advantage of performing an efficient local search and ensuring the optimal location, although they are sensitive to the initial conditions used [4]. Metaheuristic methods have the advantage of doing a good global search (search space exploration) but do not guarantee optimal solutions [5].

In this work, the adjustment of stabilizers in multimachine systems is formulated as an optimization problem aiming at maximizing the damping coefficient of the dominant eigenvalue of the closed-loop system. In order to ensure the robustness of PSS, a set of operating conditions at the tuning phase is considered. Simulations are performed using the
MATLAB $^{\circledR}$ platform, and the system is represented by state space linearized equations [6].

As a contribution, to solving the optimization problem, this paper presents a hybrid methodology based on the ABC (Artificial Bee Colony) metaheuristic proposed in [7] and a method based on objective function derivatives (QuasiNewton method - Broyden, Fletcher, Goldfarb and ShanoBFGS [4]). The goal is to couple the inherent advantages of each method: the good global search capability of the ABC metaheuristic with the local search capability of the QuasiNewton BFGS method.

The methodology is applied to the New England test system, which is well known in the literature [8]. The performance of the proposed approach outperforms the performance of some methods from the literature like the conventional ABC, Particle Swarm Optimization (PSO), and Genetic Algorithm (GA).

\section{PROBLEM PRESENTATION}

\section{A. Open Loop System Model (without PSS)}

The equations that describe the system's dynamic behavior are linearized since the interest is the analysis of stability to small perturbations [6]. Equation (1) describes the open-loop system.

$$
\begin{aligned}
& \dot{x}=A \cdot x+B \cdot u \\
& y=C \cdot x+D \cdot u
\end{aligned}
$$

in which $x$ are the state variables (internal voltages, terminal speeds, internal angles, and field voltages), $u$ represents the input variables (mechanical power and automatic voltage regulator reference) and $y$ are the output variables (angular velocity). $A, B, C$ and $D$ are respectively the state, input, output and direct transmission matrices in open-loop operation (without stabilizers).

\section{B. Closed Loop System (with PSS)}

After including the controllers through a feedback process, the state-space model in equation (2) is obtained.

$$
\begin{aligned}
\dot{x}_{M F} & =A_{M F} \cdot x_{M F}+B_{M F} \cdot u \\
y & =C_{M F} \cdot x+D_{M F} \cdot u
\end{aligned}
$$

in which $x_{M F}$ is the vector of closed-loop state variables that, in addition to the open-loop system variables $x$ (in (1)), also includes controller state variables.

The stability of the closed-loop system can be assessed from the eigenvalues of $A_{M F}$ [6][8]. 


\section{Controller Structure}

The feedback process is made as it shows in the Fig.1.

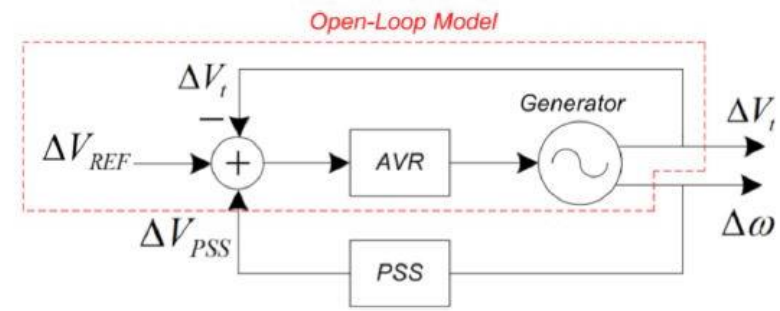

Fig. 1. Closed-loop system.

The classic controller structure used in the work is presented in (3) [3].

$$
\operatorname{ESP}(s)=K_{P S S} \times \frac{\left(1+s \frac{\sqrt{\alpha_{p}}}{\omega_{p}}\right)^{n b}}{\left(1+s \frac{1}{\omega_{p} \cdot \sqrt{\alpha_{p}}}\right)^{n b}} \times \frac{\left(s . T_{w}\right)}{\left(1+s . T_{w}\right)}
$$

in which $T_{w}$ is the time constant of the washout filter used for the stabilizer to act only in the transient regime (this constant is known), $K_{P S S}$ is a static gain, $\alpha_{p}$ and $\omega_{p}$ are the phase compensation parameters. The parameters to be adjusted for each controller are the gain $K_{P S S}$ and the phase constants $\alpha_{p}$ and $\omega_{p}[2]$.

\section{Optimization Problem}

The tuning procedure is formulated as an optimization problem to maximize the damping coefficient of the closedloop system eigenvalues by considering a set of predefined operating points to ensure robustness.

The objective function of the problem, defined in (4), corresponds to the minimum damping $\xi_{\text {min }}$ obtained considering all closed-loop eigenvalues under all operating conditions.

$$
\left\{\begin{array}{cc}
\text { Min } & -\xi_{\min } \\
& K_{P S S p}^{\min } \leq K_{P S S p} \leq K_{P S S p}^{\max } \\
\text { s.a } & \alpha_{p}^{\min } \leq \alpha_{p} \leq \alpha_{p}^{\max } \\
& \omega_{p}^{\min } \leq \omega_{p} \leq \omega_{p}^{\max }
\end{array}\right.
$$

It is desired to maximize the damping coefficient of the closed-loop dominant eigenvalue: however, in this work, the optimization algorithms are formulated to minimize functions. In this case, the negative sign used is justified.

The structure of an individual $x_{i}$ is given in (5), for an illustrative case of 2 generators (2 PSSs).

$$
x_{i}=\left[\begin{array}{llllll}
K_{P S S 1} & K_{P S S 2} & \alpha_{1} & \alpha_{2} & \omega_{1} & \omega_{2}
\end{array}\right]
$$

\section{PROPOSED ALGORITHM}

\section{A. Conceptualization of the proposed algorithm}

The Artificial Bee Colony algorithm - ABC is based on food collection and was proposed in [6]. The emergence of collective intelligence from bee swarms affects three essential components (food sources, employed forages, and unemployed forages) and defines two main modes of bee colony behavior (recruitment for a food source and abandonment of a source).

Initially, in order to select a food source, a foraging bee evaluates various properties related to the food source, such as its proximity to the hive, energy richness, taste of its nectar, and the ease or difficulty of extracting that energy source [9].

An Employed Forage is employed in a specific food source it is currently exploring. It carries information about this particular source and shares it with other bees waiting in the hive. Information includes the distance, direction and profitability of the food source. Already a foraging bee looking for a food source to exploit is called unemployed and may be an explorer randomly researching the environment or a bystander trying to find a food source through the information given by the employed bee. The average number of explorers is about 5 to $10 \%$.

The most important occurrence in the formation of collective knowledge is the exchange of information between bees. The main part of the hive regarding information exchange is the dance area. It is in this area that communication occurs between bees related to the quality of food sources. Because information about all current rich sources is available to a dance floor spectator, a spectator could probably watch various dances and choose to employ the most lucrative source. Viewers are more likely to choose more profitable sources as more information about the most profitable sources circulates. The employed foragers share their information with a probability that is proportional to the profitability of the food source, and the sharing of this information through dance is longer in duration. Thus, recruitment is proportional to the profitability of a food source [7].

\section{B. ABC Algorithm}

In the $\mathrm{ABC}$ algorithm, the position of a food source represents a possible solution to the optimization problem and the amount of nectar from a food source corresponds to the quality (suitability) of the associated solution. Initially, in the hive, $50 \%$ of the bees are employed and the other half are spectator bees [7][9].

Each cycle (iteration) research consists of three stages: (I) In the first stage of the cycle, the employed bees enter the hive and share information about the source nectar with the spectating bees waiting in the dance area. After sharing their information with the spectators, all the employed bees go to the area of the food source visited by her in the previous cycle and then choose a new food source through visual information in the vicinity and evaluate her nectar content. (II) In the second step, a spectator bee prefers a food source area depending on the nectar information distributed by the bees employed in the dance area. As the amount of nectar from a food source increases, the probability of that food source being chosen also increases. After reaching the selected area, she chooses a new food source in the vicinity of the one in 
memory, depending on the visual information, such as the bees employed. (III) In the third stage of the cycle, when nectar from a food source is abandoned by the bees, a new food source is randomly determined by an exploiting bee and replaced by the abandoned one. In the ABC model, each cycle at most one scout goes out to look for a new food source, and the number of employed and observing bees is selected to be equal to each other. These three steps are repeated until the maximum number of generations or until a stopping criterion is met [7][9].

A food source is chosen by a spectator bee depending on the probability value associated with that food source, $P_{i}$, as shown in (6):

$$
p_{i}=\frac{f i t_{i}}{\sum_{n=1}^{S N} f i t_{n}},
$$

Where fit $_{i}$ is the value of aptitude in solution $i$ and $S N$ is the number of food sources (number of individuals) equal to the number of employed bees or bystander bees.

$\mathrm{ABC}$ uses equation (7) to produce a candidate food source position from the old memory.

$$
v_{i j}=x_{i j}+\phi_{i j}\left(x_{i j}-x_{k j}\right)
$$

in which $k \in\{1,2, \ldots, S N\}$ and $j \in\{1,2, \ldots, D\}$ ( $D$ is the number of optimization parameters) are randomly chosen indices and have to be different from $i . \phi_{i j}$ is a random number between $[-1,1]$.

In $\mathrm{ABC}$, if a position cannot be improved through a predetermined number of cycles, it is assumed that this food source will be abandoned by the bees and replaced with a new food source by the scouts. The dropout limit given in (8) is the value of the predetermined number of cycles and an important control parameter of the $\mathrm{ABC}$ algorithm.

$$
x_{i}^{j}=x_{\min }^{j}+\operatorname{rand}[0,1]\left(x_{\max }^{j}-x_{\min }^{j}\right)
$$

After each candidate source position $v_{i j}$ is produced and then evaluated by the artificial bee, its performance is compared to its former one. If the new food source has nectar equal to or better than the old source, it will be replaced by the old one in memory. Otherwise, the old one is retained in memory. That is, a greedy selection mechanism is employed.

\section{Proposed Algorithm}

The proposed modification is divided into two stages and

\begin{tabular}{|c|c|}
\hline Hybrid ABC Algorithm & Step \\
\hline Start & 1 \\
\hline $\begin{array}{l}\text { Define the objective function and the problem } \\
\text { variables and initialize the algorithm parameters. }\end{array}$ & 2 \\
\hline Initialize the solution population & 3 \\
\hline Evaluate the solutions & 4 \\
\hline The best food source is memorized & 5 \\
\hline Cycle $=1$ & 6 \\
\hline Repeat & 7 \\
\hline $\begin{array}{l}\text { Produce new solutions for employed bees using (7) and } \\
\text { evaluate them }\end{array}$ & 8 \\
\hline $\begin{array}{l}\text { Apply the greedy selection process to the employed } \\
\text { bees }\end{array}$ & 9 \\
\hline Calculate Probability Values $P_{i}$ for solutions $x_{i}$ per (6) & 10 \\
\hline $\begin{array}{l}\text { Produce the new solutions } v_{i} \text { for solution viewers } x_{i} \\
\text { selected depending on the } P_{i} \text { and evaluate them }\end{array}$ & 11 \\
\hline Apply the greedy selection process to viewers & 12 \\
\hline $\begin{array}{l}\text { Determine the abandoned solution for the observer, if } \\
\text { any, and replace it with a new randomly produced } \\
\text { solution } x_{i} \text { per (8) }\end{array}$ & 13 \\
\hline Memorize the best solution achieved so far & 14 \\
\hline $\begin{array}{l}\text { Every } 20 \text { cycles do } \\
\text { Run the BFGS method from the optimal point } \\
\text { The method must be performed to refine stabilizer } \\
\text { gains. The phase adjustment is fixed at the values of the } \\
\text { best food source found so far. }\end{array}$ & 15 \\
\hline Cycle $=$ cycle +1 & 16 \\
\hline End-Repeat & 17 \\
\hline $\begin{array}{l}\text { Run the BFGS method from the optimal point } \\
\text { The method must be performed to refine all stabilizer } \\
\text { adjustment parameters (gain and phase). }\end{array}$ & 18 \\
\hline End & 19 \\
\hline
\end{tabular}
corresponds to the sixteenth and nineteenth steps in the proposed hybrid algorithm, presented in Fig. 2.
Fig. 2. Proposed Hybrid Algorithm.

In the first stage (15th step, Fig.2) a Quasi-Newton optimization method (BFGS) based on 1st and 2nd order derivatives of the objective function is performed for the refinement of the gain parameters $\left(K_{P S S}\right)$ keeping the phase constants $\left(\alpha_{p}\right.$ and $\left.\omega_{p}\right)$ fixed at the value of the best position of a food source. The BFGS method runs every 20 generations of the ABC method, considering the best position of a food source as the initial condition.

The second stage begins after $\mathrm{ABC}$ convergence, in step 18, the same Quasi-Newton optimization method (BFGS) is performed for simultaneous refinement of gain parameters $\left(K_{P S S}\right)$ and phase compensation $\left(\alpha_{p}\right.$ and $\left.\omega_{p}\right)$.

\section{APPLICATION OF THE PROPOSED METHODOLOGY}

To validate the proposed methodology, the New England system (Pai, 1989) is used. The simulations were performed using an Intel Core i3 $1.80 \mathrm{GHz}$ computer with 6 GB RAM and 64-bit Windows 7 operating system.

\section{A. System Description and Operating Points}

The New England test system, described in Fig. 3, consists of 10 generators and 39 bars. All units are equipped with stabilizers except the machine at bar 39, which was modeled as an infinite bus (equivalent system). System data can be found at (Pai, 1989). 


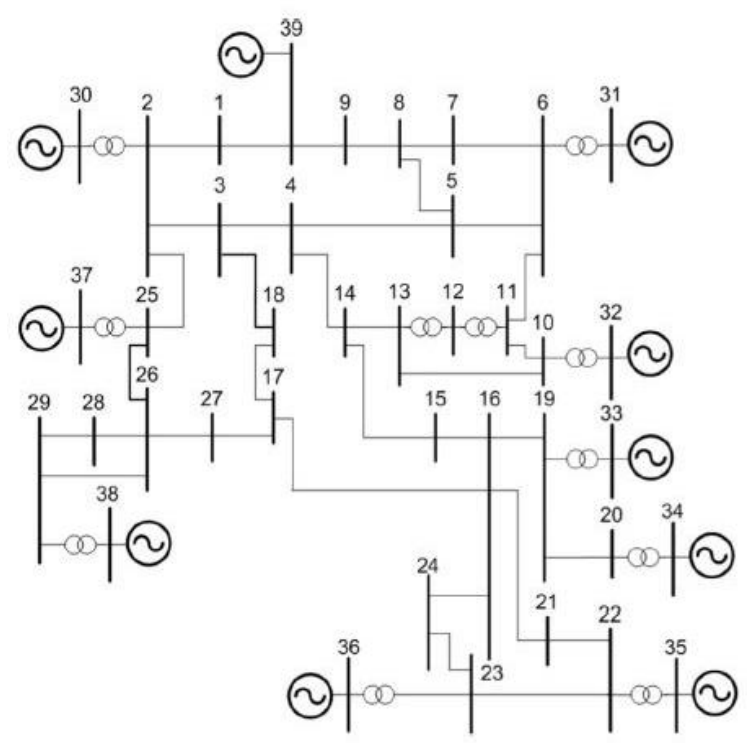

Fig. 3. New England System

The objective is to apply the proposed hybrid $\mathrm{ABC}$ method to the design of the nine stabilizers. The results will be compared with those provided by the ABC (original), PSO and AG methods. Table I shows four pre-specified operating conditions obtained from [3].

TABLE I. OPEN-LOOP OPERATING CONDITIONS

\begin{tabular}{|c|c|c|}
\hline Case & Configuration & $\begin{array}{c}\text { Minimal } \\
\text { damping (\%) }\end{array}$ \\
\hline 1 & Base Case & 1,53 \\
\hline 2 & Lines 3-18 and 25-26 out of service & $-9,59$ \\
\hline 3 & $+20 \%$ of charge & $-8,76$ \\
\hline 4 & $-20 \%$ of charge & 1,57 \\
\hline
\end{tabular}

It is observed that the open-loop system (without the controllers) is unstable in scenarios 2 and 3. State-space matrices for the various operating scenarios were obtained through the software PacDyn developed by CEPEL.

\section{B. Parameter Definition}

Table II shows the control parameter limits used in the tuning process, and the values of the best initial population solution in all methods (which corresponds to a minimum 5\% closed-loop damping). Three compensation blocks and a time constant of $5 \mathrm{~s}$ for the washout filter are considered [3][10].

\section{TABLE II. CONTROL PARAMETER Limits}

\begin{tabular}{|c|c|c|c|}
\hline $\begin{array}{c}\text { Controller } \\
\text { Parameter }\end{array}$ & Lower & Upper & Initial \\
\hline$K_{P S S}$ & 0 & 20 & 19 \\
\hline$\alpha_{P}$ & 0,1 & 10 & 8 \\
\hline$\omega_{P}$ & $0,4 \pi$ & $4 \pi$ & 10 \\
\hline
\end{tabular}

The parameters used for the $\mathrm{ABC}$ methods and the proposed methodology are: population composed of 50 individuals and 100 generations as a convergence criterion. For the ABC method, an abandonment limit parameter of 100 cycles (value of the predetermined number of cycles and an important control parameter of the $\mathrm{ABC}$ algorithm) is used.

For the PSO and AG methods, the same number of generations and individuals was used in the population. In addition, for PSO, acceleration constants equal to $\mathrm{c}_{1}=2$ and $\mathrm{c}_{2}=2$ were used. For AG, crossing and mutation probabilities equal to $60 \%$ and $5 \%$, respectively, were used.

For each methodology, fifteen simulations were carried out in order to assess the quality of the solutions

\section{Results}

The results obtained (minimum damping) using the proposed methodology are shown in Table III. It is possible to notice that the proposed approach presented better results. The increase in computational time is not an impediment, since the adjustment is not made in real-time [6].

TABLE III. RESULTS

\begin{tabular}{|c|c|c|c|c|}
\hline \multirow{2}{*}{ Method } & \multicolumn{3}{|c|}{ Minimal Cushioning (\%) } & Average time \\
\cline { 2 - 4 } & Worst & Average & Better & \\
\hline Proposed & 21,14 & 23,56 & 26,34 & 74,43 \\
\hline PSO & 14,09 & 22,80 & 26,01 & 32,80 \\
\hline ABC & 14,67 & 19,61 & 23,81 & 62,88 \\
\hline AG & 12,25 & 18,56 & 22,07 & 33,54 \\
\hline
\end{tabular}

Fig. 4 shows the box diagram (boxplot) elaborated with the results obtained in fifteen simulations (minimum damping in the four closed-loop scenarios). It is possible to notice that the proposed approach has reduced the variability of the results. The red line in the boxes (second quartile or median) median indicates that $50 \%$ of the results are greater than their value. The proposed method managed to improve significantly compared to the original $\mathrm{ABC}$. The upper blue line in the boxes in Fig. 4 (third quartile) indicates that $25 \%$ of the results obtained are higher than its value: for the proposed method it is equal to $24.09 \%, 25.72 \%$ for the PSO, $21.30 \%$ for $\mathrm{ABC}$, and $20.19 \%$ for AG. This expresses that the proposed method offers better results than the $\mathrm{ABC}$ and $\mathrm{AG}$ methods. It is observed that the results obtained by the $\mathrm{ABC}$, PSO, and AG methods are spread over a wide range of values and this problem can be solved by improving the local research capacity at $\mathrm{ABC}$.

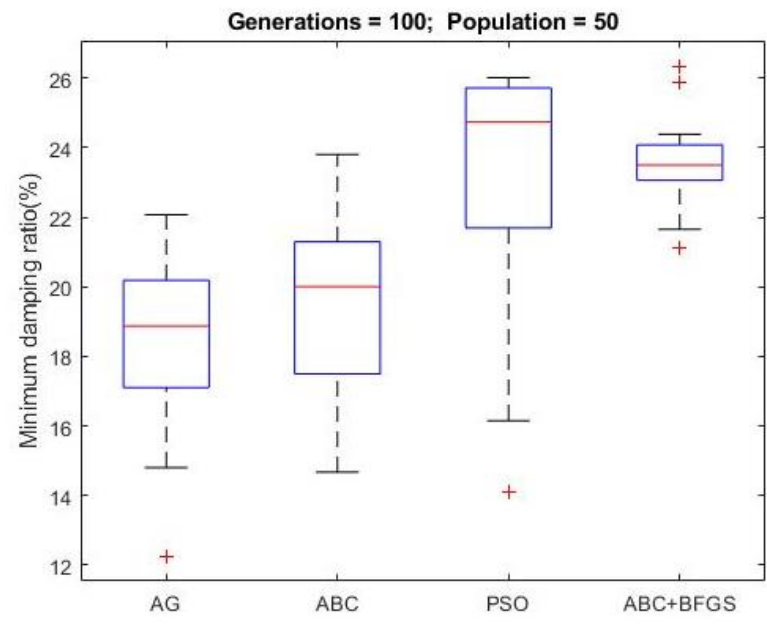

Fig. 4. Comparison of solutions: box diagrams 


\section{Comparison of solutions: box diagrams}

Considering the best solutions in Table III, Table IV presents the damping in each operating scenario. The unstable scenarios (in the open-loop), and with the lowest damping factors (in closed-loop) are highlighted.

TABLE IV. MINIMUM CUSHIONING

\begin{tabular}{|c|c|c|c|c|c|}
\hline Scenario & \multirow{2}{*}{$\begin{array}{c}\text { Without } \\
\text { PSS }\end{array}$} & \multicolumn{4}{|c|}{ With PSS } \\
\cline { 3 - 6 } & 1,53 & 26,97 & 25,04 & 27,84 & 23,41 \\
\hline 1 & $-9,59$ & 26,85 & 24,92 & 27,82 & 24,08 \\
\hline 2 & $-8,76$ & 26,34 & 23,81 & 26,07 & 22,07 \\
\hline 3 & 1,57 & 27,02 & 23,86 & 26,01 & 22,13 \\
\hline 4 & & \multicolumn{4}{|c}{} \\
\hline
\end{tabular}

It is observed that the designed control system is robust, with adequate damping factors in all operating scenarios. The parameters of the controllers obtained by the various methodologies (considering the best solutions) are shown in Table V.

TABLE V. CONTROLLERS PARAMETER

\begin{tabular}{|c|c|c|c|c|c|c|}
\hline \multirow{2}{*}{$\begin{array}{c}\text { Gener } \\
\text { ator }\end{array}$} & \multicolumn{3}{|c|}{ Proposed } & \multicolumn{3}{c|}{ ABC } \\
\cline { 2 - 7 } & $\mathbf{K}_{\mathbf{P S S}}$ & $\boldsymbol{\alpha}_{\mathbf{P}}$ & $\boldsymbol{\omega}_{\mathbf{P}}$ & $\mathbf{K}_{\mathbf{P S S}}$ & $\boldsymbol{\alpha}_{\mathbf{P}}$ & $\boldsymbol{\omega}_{\mathbf{P}}$ \\
\hline 30 & 18,84 & 9,81 & 4,26 & 19,79 & 10,00 & 4,64 \\
\hline 31 & 17,33 & 7,44 & 5,47 & 20,00 & 10,00 & 6,20 \\
\hline 32 & 18,17 & 8,89 & 5,72 & 20,00 & 9,87 & 6,42 \\
\hline 33 & 18,22 & 8,67 & 6,58 & 20,00 & 8,52 & 6,97 \\
\hline 34 & 19,02 & 7,70 & 6,91 & 17,68 & 10,00 & 7,36 \\
\hline 35 & 18,16 & 6,50 & 5,48 & 18,07 & 8,59 & 4,65 \\
\hline 36 & 19,14 & 8,52 & 9,17 & 20,00 & 7,69 & 10,42 \\
\hline 37 & 18,05 & 9,21 & 5,37 & 20,00 & 9,92 & 7,21 \\
\hline 38 & 19,47 & 8,84 & 11,72 & 20,00 & 8,53 & 10,00 \\
\hline Gener & & & & $\mathbf{A G}$ & \\
\cline { 2 - 8 } ator & $\mathbf{K}_{\mathbf{P S S}}$ & $\boldsymbol{\alpha}_{\mathbf{P}}$ & $\boldsymbol{\omega}_{\mathbf{P}}$ & $\mathbf{K}_{\mathbf{P S S}}$ & $\boldsymbol{\alpha} \mathbf{P}$ & $\boldsymbol{\omega}_{\mathbf{P}}$ \\
\hline 30 & 20,00 & 10,00 & 4,36 & 19,00 & 9,81 & 4,38 \\
\hline 31 & 20,00 & 10,00 & 6,70 & 19,00 & 7,82 & 5,78 \\
\hline 32 & 11,10 & 10,00 & 5,83 & 19,00 & 9,49 & 6,24 \\
\hline 33 & 20,00 & 10,00 & 8,28 & 19,00 & 8,06 & 6,34 \\
\hline 34 & 19,99 & 6,86 & 6,80 & 19,34 & 8,00 & 7,95 \\
\hline 35 & 20,00 & 10,00 & 5,68 & 19,00 & 8,00 & 4,81 \\
\hline 36 & 18,58 & 10,00 & 12,57 & 9,41 & 8,00 & 10,00 \\
\hline 37 & 15,19 & 10,00 & 5,26 & 19,31 & 8,00 & 5,91 \\
\hline 38 & 20,00 & 9,99 & 12,57 & 19,00 & 8,00 & 10,00 \\
\hline
\end{tabular}

\section{CONCLUSIONS}

This article presented a hybrid algorithm to solve the coordinated design of power system stabilizers. The adjustment procedure was formulated as an optimization problem in order to optimize the minimum system damping considering multiple operating conditions.
The proposed method associates the advantage of the global search made by the ABC method with the speed of the local search made by the Quasi-Newton (BFGS) method. The inclusion of the local search step allowed obtaining better results both in quality and in robustness (less dispersion when considering several simulations), since, when the current point is close to a minimum (local or global), search direction methods (with derivatives) manage to converge to such points.

The results obtained with the proposed methodology were superior to those obtained with the use of conventional ABC and Genetic Algorithms. When compared to the PSO (method widely used in the literature), the proposed method was superior in terms of dispersion of results (values between $21.14 \%$ to $26.34 \%$ ), whereas the PSO had a variation of $16.15 \%$ to $26.01 \%$ for the damping values.

\section{ACKNOWLEDGMENT}

The authors thank CEPEL for authorizing the use of the PacDyn program.

\section{REFERENCES}

[1] Demello, F. P., and Concordia, C. "Concepts of Synchronous Machine Stability as Affected by Excitation Control," Power Apparatus and Systems, IEEE Transactions on, 1969, v. PAS-88, n. 4, p. 316-329. ISSN: 0018-9510 DOI: 10.1109/TPAS.1969.292452.

[2] Santos, A. S., and Peres, W. "Evolucionário PSO Hibridizado com Método Hooke-Jeeves para o Projeto Robusto e Coordenado de Estabilizadores De Potência," In: CBA 2018, João Pessoa, Paraíba. XXII Congresso Brasileiro de Automática, 2018. DOI:10.20906/CPS/CBA2018-0520.

[3] Peres, W., Júnior, I. C. S. and Filho, J. A. P. "Gradient Based Hybrid Metaheuristics for Robust Tuning of Power System Stabilizers," International Journal of Electrical Power \& Energy Systems, 2018, v. 95, p. 47-72. ISSN: 0142-0615.

[4] Luenberger, D. "Linear and nonlinear programming," 2nd. ed. [S.1.]: Springer, 2003.

[5] Silva, A. P. A., and Abrao, P. J. "Applications of evolutionary computation in electric power systems," Evolutionary Computation, CEC '02. Proceedings of the 2002 Congress on. [S.1.]: [s.n.], 2002, p. 1057-1062.

[6] Kundur, P. "Power System Stability and Control," $1^{\mathrm{a}}$. ed. [S.1.]: McGraw-Hill, 1994, 1196 p.

[7] Karaboga, D. "An Idea Based on Honey Bee Swarm for Numerical Optimization," Technical report-tr06, october. Erciyes University, Engineering Faculty Computer Engineering Department Kayseri/Türkiye, 2005.

[8] Pai, A. "Energy Function Analysis for Power System Stability," Power Electronics and Power Systems, 1989. [S.1.]: Springer. ISBN: 0792390350.

[9] Karaboga, D., and Akay, B. "A comparative study of Artificial Bee Colony algorithm," Applied Mathematics and Computation 214, 2009, p. 108-132.

[10] Santos, A. S. Santos and Peres, W. "A hybrid EPSO for power system stabilizers design," 2018 Simposio Brasileiro de Sistemas Eletricos (SBSE), 2018, pp. 1-6, doi: 10.1109/SBSE.2018.8395613. 